\title{
Imprint Molding of a Microfluidic Optical Cell on Thermoplastics with Reduced Surface Roughness for the Detection of Copper Ions
}

\author{
Jing Wu and Nae Yoon LEE ${ }^{\dagger}$ \\ Department of BioNano Technology, Gachon University, 1342 Seongnam-daero, Sujeong-gu, Seongnam, \\ Gyeonggi-do 461-701, Korea
}

\begin{abstract}
Here, we introduce a simple and facile technique for fabricating microfluidic optical cells by utilizing a micropatterned polymer mold, followed by imprinting on thermoplastic substrates. This process has reduced the surface roughness of the microchannel, making it suitable for microscale optical measurements. The micropatterned polymer mold was fabricated by first micromilling on a poly(methylmethacrylate) (PMMA) substrate, and then transferring the micropattern onto an ultraviolet (UV)-curable optical adhesive. After an anti-adhesion treatment of the polymer mold fabricated using the UV-curable optical adhesive, the polymer mold was used repeatedly for imprinting onto various thermoplastics, such as PMMA, polycarbonate (PC), and poly(ethyleneterephthalate) (PET). The roughness values for the PMMA, PC, and PET microchannels were approximately $11.3,20.3$, and $14.2 \mathrm{~nm}$, respectively, as compared to those obtained by micromilling alone, which were $15.9,76.8$, and $207.5 \mathrm{~nm}$, respectively. Using the imprint-molded thermoplastic optical cell, rhodamine B and copper ions were successfully quantified. The reduced roughness of the microchannel surface resulted in improved sensitivity and reduced noise, paving the way for integration of the detection module so as to realize totally integrated microdevices.
\end{abstract}

Keywords Thermoplastics, UV-curable optical adhesive, imprint molding, optical cell, roughness reduction, detection module

(Received August 29, 2015; Accepted October 16, 2015; Published January 10, 2016)

\section{Introduction}

With growing interest in the development of microscale total analysis systems ( $\mu \mathrm{TAS})$, the fabrication of miniaturized detection modules has attracted much attention because of their considerable potential for realizing a totally integrated miniaturized device. ${ }^{1}$ Concerning miniaturized detection modules, three kinds of analytical methods have been widely adopted: electrochemical, ${ }^{2-4}$ optical, ${ }^{1,5-7}$ and mass spectrometric ${ }^{8-10}$ detection. Among these methods, optical detection allows for the fast identification of chemical concentrations with relative ease. It also provides high reliability in measurement accuracy. However, the fabrication of high-performance microfluidic optical cells remains to be a challenging issue, because any surface roughness can significantly affect the optical measurements.

For fabricating microfluidic optical cells, thermoplastics are widely utilized in place of poly(dimethylsiloxane) (PDMS) elastomer, because relatively fewer small hydrophobic molecules, such as fluorescent dyes, biomolecules, and certain drugs, are absorbed onto thermoplastics, as compared to PDMS. Also, most thermoplastics are optically transparent, lightweight, and inexpensive. When it comes to fabricating absorption cells, particularly when the detection method is the UV/vis measurement, the surface roughness of the microchannel becomes a crucial issue. ${ }^{11}$ Many techniques have been developed

$\dagger$ To whom correspondence should be addressed.

E-mail: nylee@gachon.ac.kr for engraving microchannels onto thermoplastics, such as hot embossing, ${ }^{12-14}$ laser cutting, ${ }^{15-18}$ and computer numerical control (CNC) micromilling, ${ }^{11,19,20}$ to name only a few. Among these, hot embossing is relatively simple and fast, but it requires the use of expensive metal molds. Because micropatterns on metal molds cannot be readily modified whenever a new micropattern needs to be devised, it can be a costly process. Laser cutting requires an expensive laser machine, as well as complicated maintenance facilities. Micromilling has also been widely adopted because microstructures can be directly engraved onto the surface of the substrate, and because microstructures can be easily devised by a relatively inexpensive process. Nevertheless, depending on the type of substrate, engraved microchannels could result in a rough surface.

Many efforts have been made to reduce the surface roughness of microchannels engraved onto thermoplastics. For example, Lee et al. ${ }^{20}$ deposited a $\mathrm{SiO}_{2}$ layer along engraved poly(methylmethacrylate) (PMMA) microchannels to obtain a smoother inner surface while realizing permanent sealing with PDMS assisted by the coated intermediate $\mathrm{SiO}_{2}$ layer. Huang et al. ${ }^{21}$ preheated PMMA to approximately $70-90^{\circ} \mathrm{C}$ during laser processing to reduce the surface roughness. Ogilvie et al. ${ }^{11}$ suggested a chloroform vapor treatment of micromilled PMMA and a cyclic olefin copolymer (COC) channel to irreversibly bond the microfluidic chip, while simultaneously reducing the channel surface roughness.

In this study, we introduce a simple and facile strategy for fabricating microfluidic optical cells by utilizing a micropatterned polymer mold and imprinting onto various thermoplastic substrates, resulting in the reduced surface roughness of a 
(a)

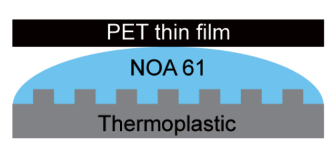

(d)

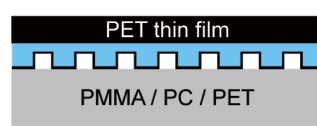

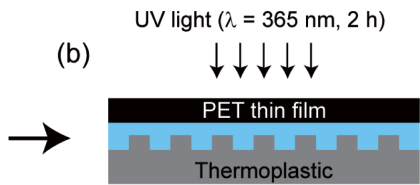

(e)

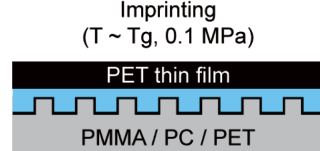

(c)
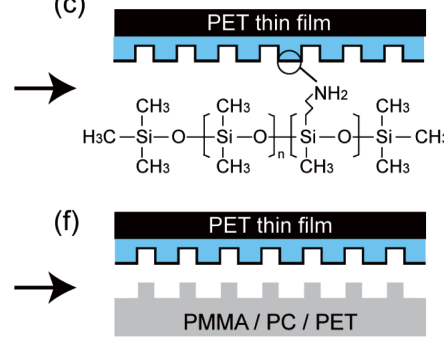

(g)

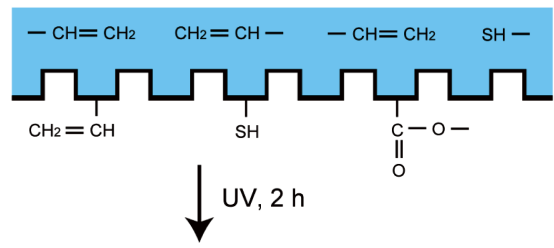

(h)

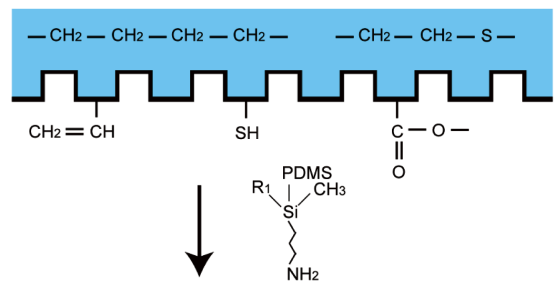

(i)

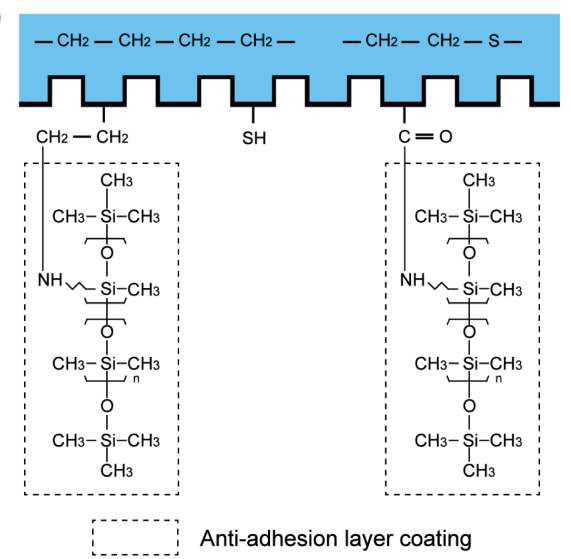

Fig. 1 Schematic showing ( $a-b)$ the fabrication of the NOA 61 polymer replica mold, (c) an anti-adhesion coating of the polymer mold, and $(\mathrm{d}-\mathrm{f})$ imprinting of the polymer mold onto PMMA, PC, and PET. (g) Chemical groups assumed to exist within NOA 61. (h) Partially UV-cured NOA 61 and surface chemical groups. (i) Amine-PDMS linker coated on a partially UV-cured NOA 61 to form anti-adhesion layer along the surface of the microchannel.

microchannel suitable for microscale optical measurements. In our previous studies, we found that, depending on the type of thermoplastic, the resulting surface roughness varied significantly, and among the widely adopted thermoplastics, PMMA offered the cleanest and smoothest surface after micromilling. Micromilled PMMA channels were thus adopted as a primary mold for transferring patterns onto optically curable prepolymers, in this case, a UV-curable adhesive, ${ }^{22}$ for the fabrication of a positive replica used as a secondary mold. After an anti-adhesion treatment of the secondary polymer mold, it was used repeatedly for imprinting onto various thermoplastics, such as PMMA, polycarbonate (PC), and poly(ethyleneterephthalate) (PET) simply by varying the temperature to more closely match the glass transition temperature $\left(T_{\mathrm{g}}\right)$ of the thermoplastic during the imprinting process. The UV-curable adhesive employed in this study was a thiolene-based UV-curable adhesive that contains thiol and acrylate functionalities. Because acrylate can directly react with the amine functionality of the amine-PDMS linker, namely, poly[dimethylsiloxane-co-(3-aminopropyl)methylsiloxane], an anti-adhesion coating can be realized under room temperature conditions. Using the imprinted thermoplastic optical cell, rhodamine B, a kind of a colorimetric dye, and copper(II) ions were detected inside the microfluidic optical cell by a UV-vis absorbance measurement.

\section{Experimental}

Reagents and chemicals

Norland Optical Adhesive (NOA) 61 (NOA 61) was purchased from Norland Company. Poly[dimethylsiloxane-co-(3-aminopropyl)methylsiloxane] (amine-PDMS linker), rhodamine B, and copper(II) sulfate pentahydrate (> 98.0\%) were purchased from Sigma-Aldrich. Isopropyl alcohol (IPA) (99.5\%) was purchased from Daejung Chemical. PDMS prepolymer (Sylgard 184) and the curing agent were purchased from Dow Corning. PMMA (thickness $2.0 \mathrm{~mm}$ ), PC (thickness $2.0 \mathrm{~mm}$ ), and PET (thickness 0.18 and $2.0 \mathrm{~mm}$ ) were purchased from Goodfellow. Microchannels were engraved using a computer numerical control (CNC) milling machine.

Fabrication of a polymer mold and imprinting onto thermoplastics Figure 1 shows the overall schematic for fabricating the polymer mold utilizing a UV-curable adhesive, NOA 61. Prior to fabricating the secondary polymer mold, a microchannel was engraved onto a thermoplastic by micromilling. The NOA 61 prepolymer was then dispensed onto the thermoplastic microchannel and pressed with a thin, flexible, plasma-treated PET film having a thickness of $18 \mu \mathrm{m}$ (Fig. 1(a)). The assembly of the NOA 61-coated PMMA and PET films was cured under UV light $(\lambda=365 \mathrm{~nm})$ (Philips, TL $8 \mathrm{~W}$ ) for $2 \mathrm{~h}$ (Fig. 1(b)). The total power of the lamp was $56 \mathrm{~W}$, and the distance between the lamp and the substrate was $6 \mathrm{~cm}$. After peeling off the partially cured NOA 61 replica from the PMMA, the 
microchannel-patterned surface was coated with an aminePDMS linker by reacting it with an undiluted amine-PDMS linker at room temperature for $20 \mathrm{~min}$ (Fig. 1(c)) for easy release of the mold after the imprinting. An unreacted aminePDMS linker solution was washed with copious amounts of IPA while sonicating for $1 \mathrm{~min}$ and dried. The anti-adhesion layercoated NOA 61 mold was then imprinted onto PMMA, PC, and PET at 120,140 , and $80^{\circ} \mathrm{C}$, respectively, at a pressure of $0.1 \mathrm{MPa}$ for $30 \mathrm{~min}$ (Figs. 1(d) - 1(f)). For a comparison, microchannels were engraved on PC and PET as well by CNC micromilling.

\section{Surface characterizations (1): Contact angle measurement}

The water contact angles were measured on a flat NOA 61 polymer mold before and after coating with an amine-PDMS linker by the sessile drop technique using a Phoenix 300 contact angle measuring system (Surface Electro Optics, Korea). The results were analyzed using Image Pro 300 software. At least five measurements were made and averaged. The water contact angle of a pristine PDMS was used as a reference.

Surface characterizations (2): X-ray photoelectron spectroscopy (XPS) analyses

XPS analyses were conducted using an Axis-Hsi (Kratos Analytical, UK) equipped with a magnesium X-ray radiation source of a dual gun $(1253.6 \mathrm{eV})$ and pass energy of $20 \mathrm{eV}$. The pressure in the chamber was below $5 \times 10^{-9}$ Torr before the data were taken, and the voltage and current of the anode were $15 \mathrm{kV}$ and $10 \mathrm{~mA}$, respectively. The take-off angle was set at $45^{\circ}$. The binding energy of $\mathrm{C} 1 \mathrm{~s}(284.5 \mathrm{eV})$ was used as the reference. The resolution for the measurement of the binding energy was about $0.1 \mathrm{eV}$.

\section{Surface characterizations (3): Atomic force microscopy (AFM)} analyses

The surface roughness of micromilled and imprinted microchannels on PMMA, PC, and PET was first measured by AFM. AFM was performed in the contact mode using a PSIA XE-150 system (PSIA Inc.) with a 1.0 scanning rate at room temperature. The scan size was set to $10 \times 10 \mu \mathrm{m}$ for surface roughness analyses by a non-contact ultra-sharp silicon cantilever (NSC 15).

\section{Surface characterizations (4): Laser light scattering}

As a second means for measuring the surface roughness, laser light scattering was performed. A straight microchannel with a width, depth, and length of $1 \mathrm{~mm}, 0.5 \mathrm{~mm}$, and $2 \mathrm{~cm}$, respectively, was micromilled on PMMA, PC, and PET, and then bonded to a homogenous flat partner substrate via thermal bonding. The light scattering was performed by utilizing an R-30993 Red HeNe Laser (Newport, US) with a 633-nm wavelength. The beam's diameter $\left(1 / \mathrm{e}^{2}\right)$ was $0.88 \mathrm{~mm}$.

\section{Detection}

The UV-vis absorbance measurement was conducted using a UV-vis spectrophotometer (Cary 50 UV-VIS-NIR, Varian) equipped with a Czerny-Turner $0.28 \mathrm{M}$ monochromator. The light was produced by a pulsed xenon lamp, which could produce scan wavelengths from 190 to $1100 \mathrm{~nm}$. The results were analyzed by Cary 50 Bio UV-vis spectrophotometer software. The experiments were conducted using a solid sample holder.

\section{Results and Discussion}

\section{Anti-adhesion coating of polymer mold}

Figures 1(g)-1(i) show the expected chemistry for the coating of the anti-adhesion layer along the microchannelpatterned surface of the polymer replica mold shown in Fig. 1(c). Figure $1(\mathrm{~g})$ shows some components anticipated to be contained in the NOA 61 prepolymer, which include vinyl, thiol, and ester functionalities. ${ }^{23}$ When the NOA 61 prepolymer was exposed under UV light for $2 \mathrm{~h}$, chemical reactions took place, possibly resulting in the formation of vinyl polymers $\left(-\mathrm{CH}_{2}-\mathrm{CH}_{2}-\mathrm{CH}_{2}-\mathrm{CH}_{2-}\right)^{24,25}$ and chemical bonds between vinyl and thiol functionalities $\left(-\mathrm{CH}_{2}-\mathrm{CH}_{2}-\mathrm{S}-\right)^{25-29}$ as intermediate components of partially cured NOA 61 polymer, as shown in Fig. 1(h). When partially cured NOA 61 reacts with the amine functionality of the amine-PDMS linker, any unreacted vinyl and ester functionalities within the partially cured NOA 61 can react with the amine functionality of the amine-PDMS linker, resulting in the formation of a urethane linkage $[-\mathrm{NH}-(\mathrm{C}=\mathrm{O})-],{ }^{30,31}$ as shown in Fig. 1(i). Vinyl and amine functionalities are also known to form a chemical bond $\left(-\mathrm{CH}_{2-}\right.$ $\left.\mathrm{CH}_{2}-\mathrm{NH}-\right)^{31-34}$ The coating performance was further verified by conducting contact angle measurements and XPS analyses.

\section{Contact angle measurements and XPS analyses}

Figure 2 shows the results of water contact angle measurements of partially cured NOA 61 (Fig. 2(a)), amine-PDMS linker-coated NOA 61 (Fig. 2(b)), and pristine PDMS bulk (Fig. 2(c)). To measure the water contact angle on a flat NOA 61 surface, it was spin coated at $3000 \mathrm{rpm}$ for $15 \mathrm{~s}$ on an oxygen plasma-treated thin PET film to obtain a homogeneous thickness, and then partially cured under UV light $(\lambda=365 \mathrm{~nm})$ for $2 \mathrm{~h}$. The water contact angle of the partially cured NOA 61 surface was approximately $63.1 \pm 0.5^{\circ}, 35$ as shown in Fig. 2(a). After coating with an undiluted amine-PDMS linker and washing with IPA and drying, the water contact angle increased to $95.3 \pm 0.9^{\circ}$, as shown in Fig. 2(b), ${ }^{31}$ whereas that of the pristine PDMS was approximately $102.9 \pm 0.9^{\circ}$, as shown in Fig. 2(c). The pristine PDMS bulk was fabricated by mixing the PDMS prepolymer with the curing agent at a ratio of 10:1 (w/w), followed by degassing and thermal curing at $80^{\circ} \mathrm{C}$ for $30 \mathrm{~min}$. These results demonstrate that the amine-PDMS linker was successfully endowed on the partially cured NOA 61. In this experiment, NOA 61 was partially cured before coating with the aminePDMS linker, so that more diverse chemical functionalities could be endowed by the chemical reaction, as compared to fully cured NOA. In our previous study, ${ }^{35}$ we measured the water contact angles on the surfaces of partially cured and fully cured NOA 61 substrates, which were approximately $61.3 \pm 3.0^{\circ}$ and $56.1 \pm 1.6^{\circ}$, respectively. The difference in the water contact angle values reflect the fact that the surface chemistry changed after full curing of the substrate. The water contact angle of the amine-PDMS-coated NOA 61 substrate was slightly lower than that of the pristine PDMS substrate, presumably because of the relatively low viscosity of the amine-PDMS linker $\left(90 \mathrm{cSt}\right.$ at $\left.25^{\circ} \mathrm{C}\right)$, as compared to that of the Sylgard 184 PDMS $\left(4000 \mathrm{mPa} \cdot \mathrm{s}\right.$ at $\left.23^{\circ} \mathrm{C}\right)$, as well as the free thiol and hydroxyl functionalities existing on the NOA 61 surface.

Figures 2(d) - 2(f) show the results of XPS analyses for the partially cured NOA 61 (Fig. 2(d)), amine-PDMS-coated NOA 61 (Fig. 2(e)), and pristine PDMS (Fig. 2(f)). For the partially cured NOA $61,{ }^{35}$ the peaks appeared at approximately 172 and $403 \mathrm{eV}$, representing the NOA characteristic peaks of S2p and N1s, respectively. After coating with the amine-PDMS linker, 


\begin{tabular}{cccccc} 
& Partially cured NOA 61 & & Amine-PDMS-coated NOA 61 & Pristine PDMS \\
\hline (a) & $63.1 \pm 0.5^{\circ}$ & (b) & $95.3 \pm 0.9^{\circ}$ & (c) & $102.9 \pm 0.9^{\circ}$ \\
\hline
\end{tabular}
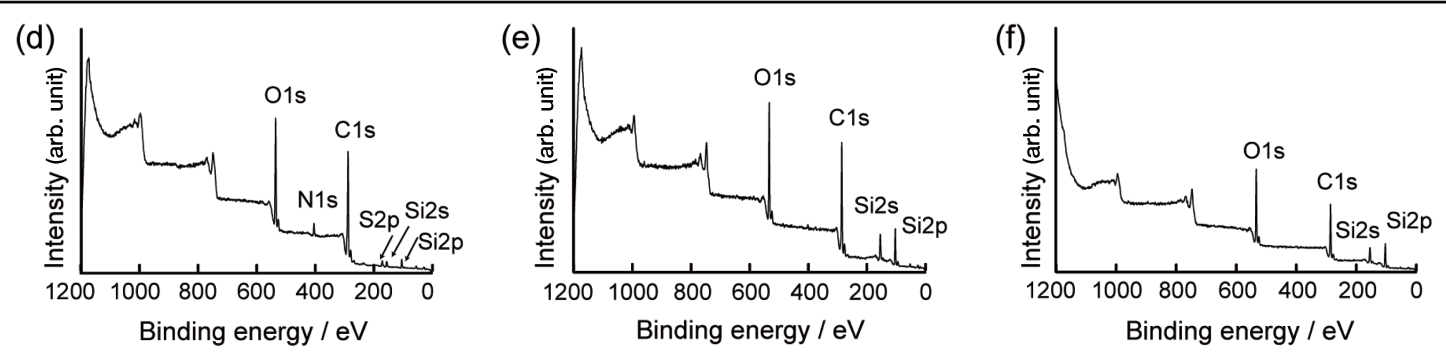

Fig. 2 (a - c) Results of water contact angle measurements and (d-f) XPS analyses for $(a, d)$ partially cured NOA 61, (b, e) amine-PDMS linker-coated NOA 61, and (c, f) pristine PDMS.
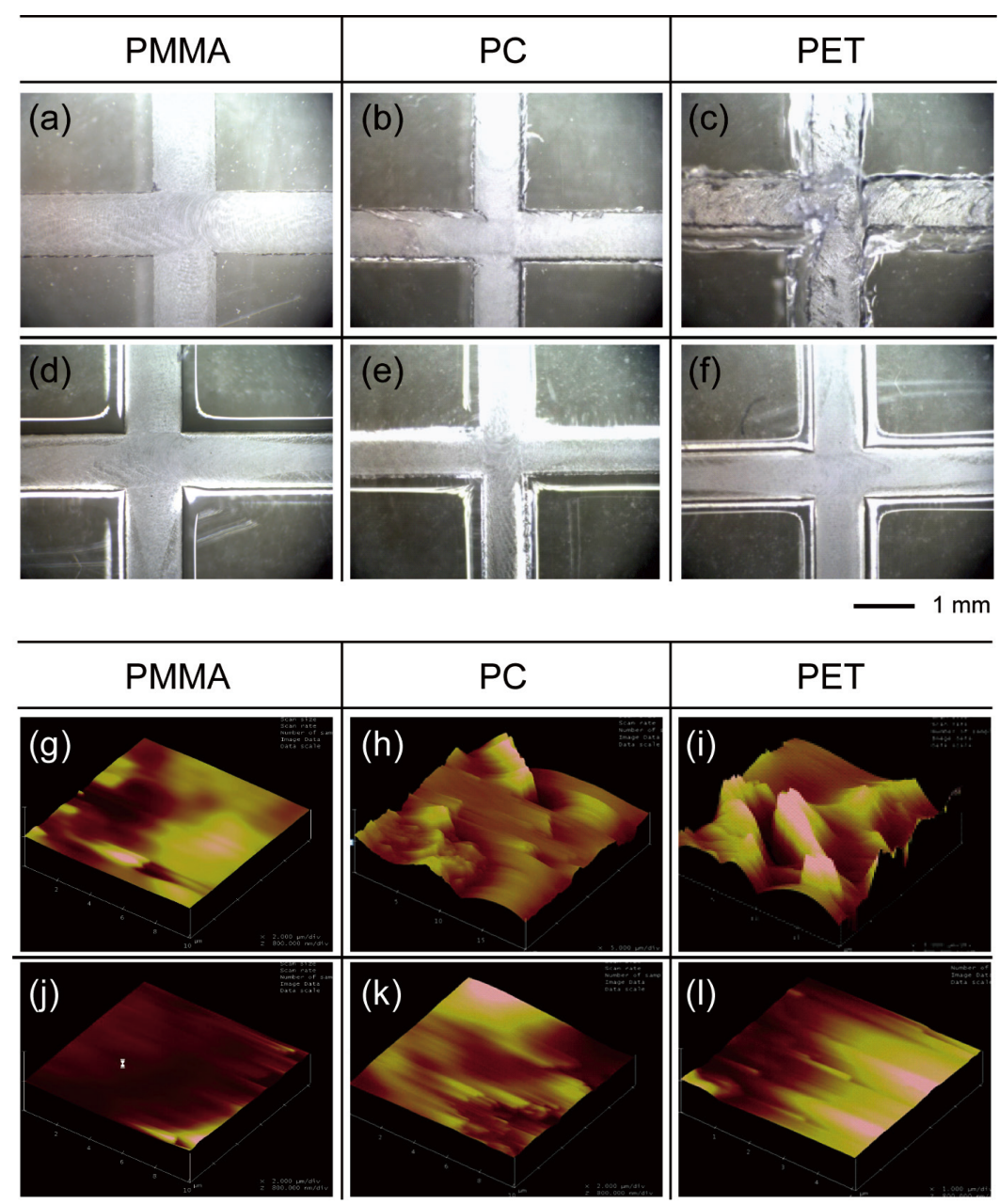

Fig. 3 Optical microscopic (OM) images showing the (a) micromilled PMMA, (b) micromilled PC, (c) micromilled PET, (d) imprint-molded PMMA, (e) imprint-molded PC, and (f) imprint-molded PET. Results of AFM analyses for (g) micromilled PMMA, (h) micromilled PC, (i) micromilled PET, (j) imprint-molded PMMA, (k) imprint-molded PC, and (l) imprint-molded PET.

however, the S2p and N1s peaks disappeared and only O1s, $\mathrm{C} 1 \mathrm{~s}, \mathrm{Si} 2 \mathrm{~s}$, and $\mathrm{Si} 2 \mathrm{p}$ peaks were detected at approximately 534, 286, 154, and $103 \mathrm{eV}$, respectively (Fig. 2(e)). The same results were found for the pristine PDMS, as shown in Fig. 2(f). The disappearance of the $\mathrm{S} 2 \mathrm{p}$ peak was probably because the coating of the NOA 61 surface with the amine-PDMS linker masked the characteristic peak of NOA 61, which contained mercapto functional groups. The reason for the appearance of the N1s peak might be due to an unknown component of the NOA 61, which could not be identified, owing to a policy of the 
(a)

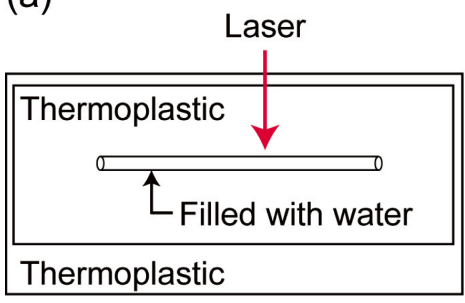

Microchannel-engraved thermoplastic
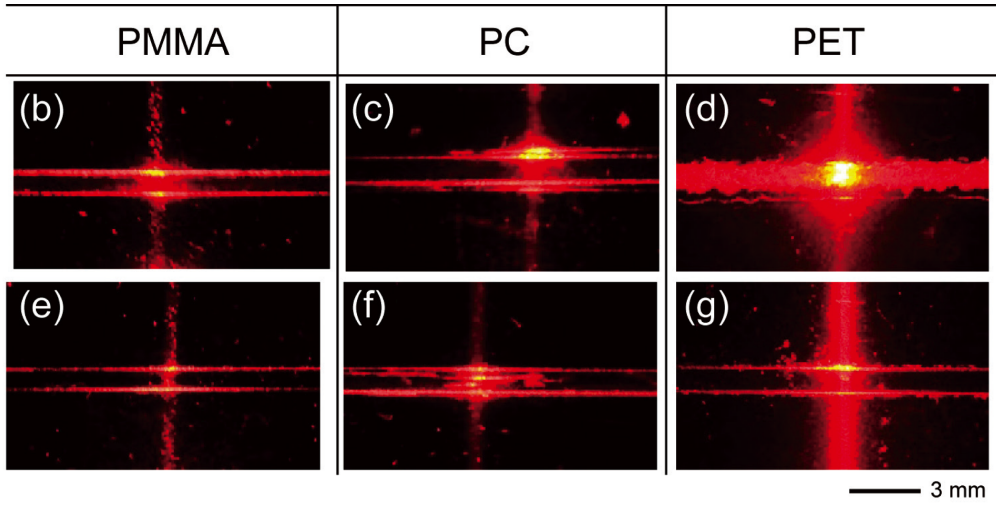

(h)
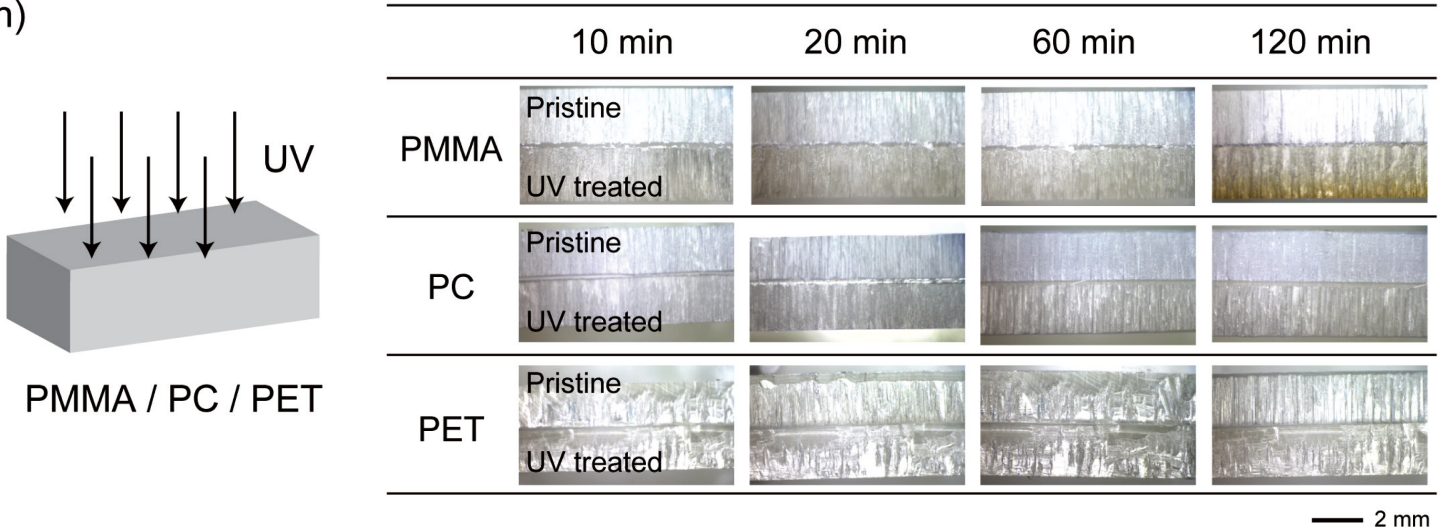

PMMA / PC / PET

Fig. 4 Results of light-scattering experiments. (a) Schematic showing the microdevice, (b) micromilled PMMA microchannel, (c) micromilled PC microchannel, (d) micromilled PET microchannel, (e) imprint-molded PMMA microchannel, (f) imprint-molded PC microchannel, and (g) imprint-molded PET microchannel. (h) Results of substrate color changes when illuminated with UV light for 10, 20, 60, and $120 \mathrm{~min}$.

manufacturer. The similarities in the appearance of the elemental peaks in Figs. 2(e) and 2(f) reflect the fact that the surface was successfully coated with the amine-PDMS linker.

\section{Surface roughness analyses (1): Optical measurements}

Figures 3(a) - 3(c) show optical microscopic (OM) images of the microchannels (width: $1 \mathrm{~mm}$, depth: $0.5 \mathrm{~mm}$ ) engraved on PMMA, PC, and PET, respectively, which were obtained by $\mathrm{CNC}$ micromilling operating under the same conditions. Based on the above results, the surface of the PMMA microchannel was the smoothest among the three thermoplastics tested. For this reason, micromilled PMMA was selected as the primary mold for obtaining a secondary polymer mold, the process of which is demonstrated in Fig. 1. Figures 3(d) - 3(f) show OM images of the microchannels obtained after imprint molding onto PMMA, PC, and PET, respectively, using the secondary polymer mold. The OM images show that there is a notable improvement, particularly in the roughness for PC and PET. The NOA 61 mold was used repeatedly over ten times without damaging the mold.

\section{Surface roughness analyses (2): AFM analyses}

Figures 3(g)-3(i) show the results of AFM analyses performed after micromilling and imprint molding. The roughness value is defined by the average spacing between waviness peaks. Specifically, if the spacing is large, the surface is considered to be rough, but if the spacing is small, the surface is considered to be smooth. The average surface roughness values for micromilled PMMA, PC, and PET were 15.9, 76.8, and $207.5 \mathrm{~nm}$, respectively. Because the PMMA surface was the smoothest, the NOA 61 prepolymer was poured onto the micromilled PMMA to produce a secondary polymer mold. As shown in Figs. 3(j) - 3(1), the roughness values of the microchannels formed on PMMA, PC, and PET after imprint molding were $11.3,20.3$, and $14.2 \mathrm{~nm}$, respectively. The roughness for PMMA, PC, and PET was reduced by 28.9, 73.6, and $93.2 \%$, respectively, which indicates that this method is effective, particularly for fabricating microchannels on PC and PET. A further roughness reduction for PMMA after the imprint molding was probably because the plasticized thermoplastic could not precisely mimic every single detail of the roughness of the secondary polymer mold during the thermal imprint molding. The AFM analyses results corresponded well with the OM images shown in Figs. 3(a) - 3(f).

\section{Surface roughness analyses (3): Light scattering measurement}

To examine any improvement of the surface roughness further, light-scattering experiments were performed inside a straight microchannel (width, $1 \mathrm{~mm}$; depth, $0.5 \mathrm{~mm}$ ) filled with distilled water. Laser light $(\lambda=633 \mathrm{~nm})$ was passed perpendicular to the microchannel. Figure 4(a) shows a schematic of the microchannel. Figures 4(b) - 4(d) show the results of light scattering experiments performed inside micromilled PMMA, PC, and PET, respectively. For PET, incident light was randomly scattered inside the microchannel; as a result, the thickness of the outlet light beam decreased as the laser light passed through the microchannel (Fig. 4(d)). Figures 4(e) - 4(g) show light scattering experiments performed inside the imprint-molded 
(a) Image and positioning of the microfluidic PET optical cell
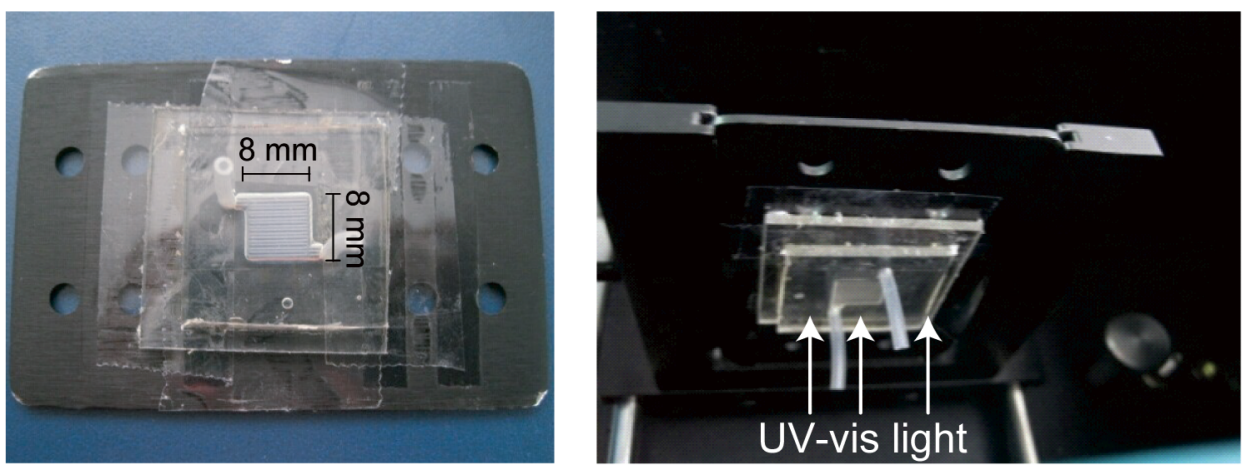

(b) Imprint-molded PET optical cell
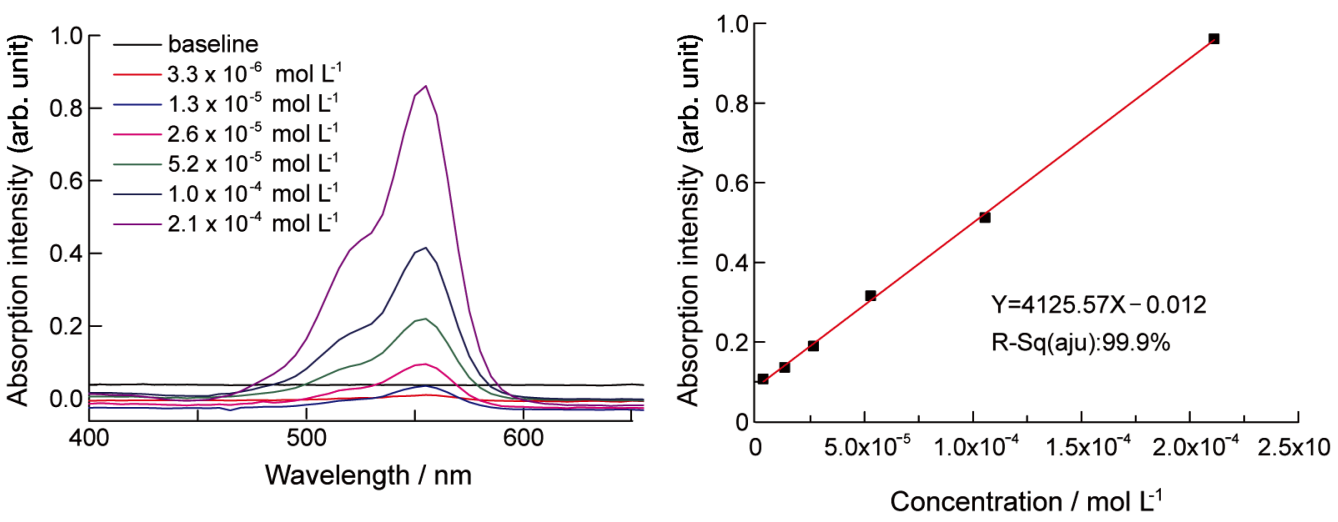

(c) Micromilled PET optical cell
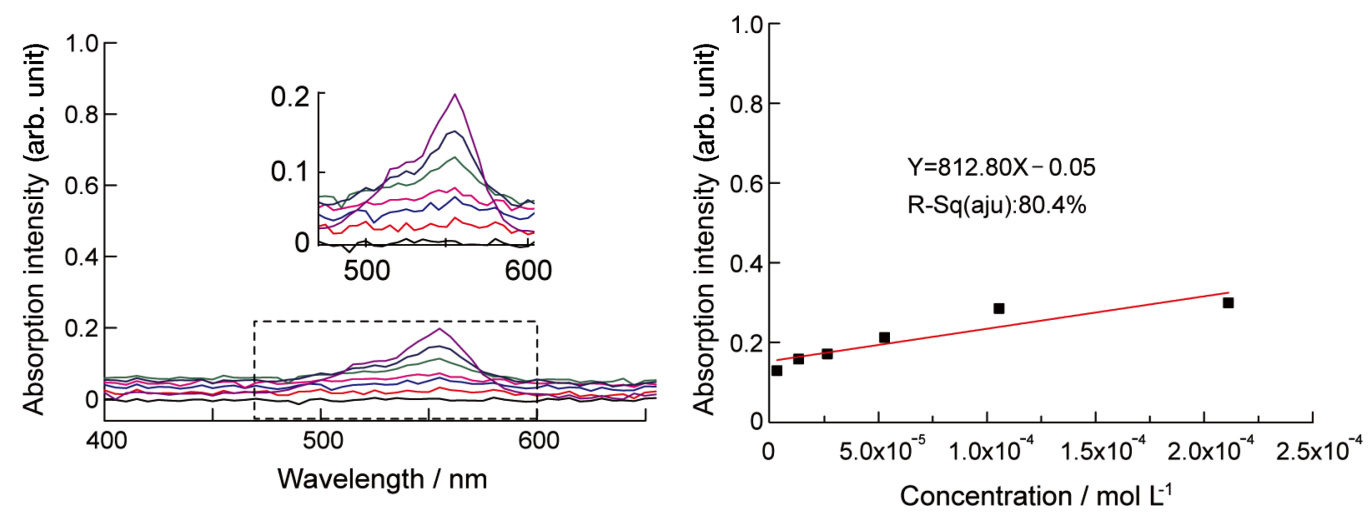

Fig. 5 Results of rhodamine B detection performed inside an imprint-molded PET using a UV-vis absorption spectrometer. (a) Photos of the PET microdevice and its positioning on the holder. (b) Absorption intensities of rhodamine B detected inside imprint-molded PET as a function of wavelength and concentration. (c) Absorption intensities of rhodamine B detected inside micromilled PET as a function of wavelength and concentration.

PMMA, PC, and PET, respectively. As demonstrated in Figs. 4(b) - 4(g), light scattering notably decreased inside the imprint-molded thermoplastics. In particular, almost all of the laser light was collected on the other side of the microchannel without much of a decrease in its output intensity as it passed through the PET microchannel (Fig. 4(g)). These results demonstrating a roughness improvement were in accordance with those shown in Fig. 3.

\section{Selection of microdevice material}

The fabrication of a microchannel with less scattering is critical when performing absorption-based detection inside a microdevice. Based on the results of the surface characterization, we selected the imprint-molding method to fabricate a microfluidic optical cell. Moreover, the microchannel needs to be transparent over long periods of UV illumination to be suitable as an optical cell and for recyclability. To examine the effect of UV illumination on the color changes of the substrates, PMMA, PC, and PET were illuminated with UV light 


\section{(a) Imprint-molded optical cell}

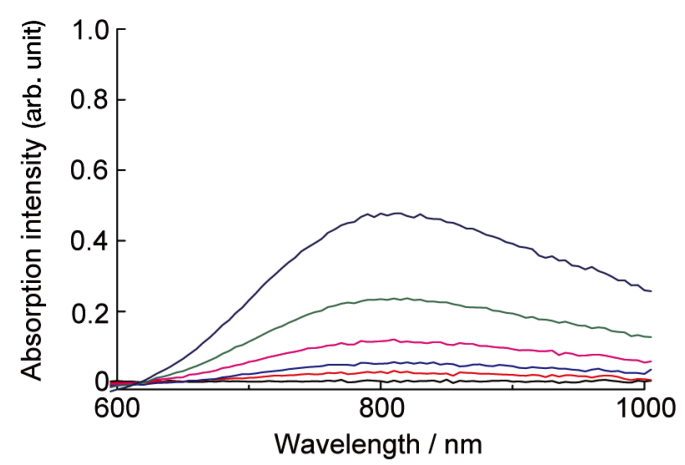

(b) Micromilled optical cell

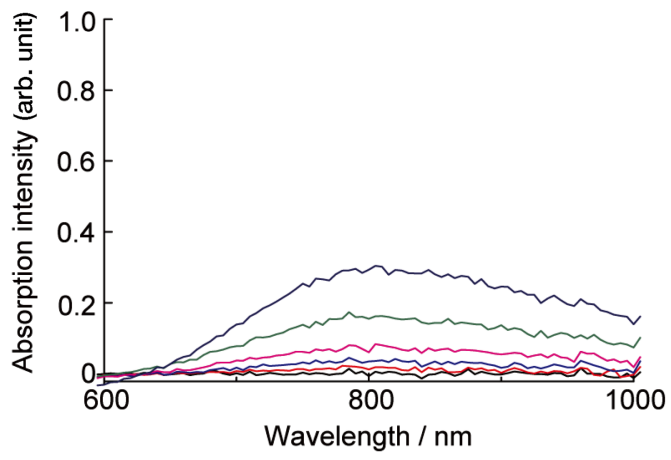

$-0.8 \mathrm{~mol} \mathrm{~L}^{-1} \longrightarrow 0.4 \mathrm{~mol} \mathrm{~L}^{-1} \longrightarrow 0.2 \mathrm{~mol} \mathrm{~L}^{-1}$

$0.1 \mathrm{~mol} \mathrm{~L}^{-1}$

$0.05 \mathrm{~mol} \mathrm{~L}^{-1}$

Baseline

Fig. 6 Results showing the detection of copper ions inside imprint-molded and micromilled PET microfluidic optical cells.

$(\lambda=365 \mathrm{~nm})$ for 10, 20,60, and $120 \mathrm{~min}$. Figure 4(h) compares the color of a pristine substrate with those illuminated with UV for a predetermined period of time. As shown in Fig. 4(h), PMMA started to change color after illumination for just 10 min. Although it occurred at a slower rate, the PC also changed color gradually. However, the PET did not change color, even after UV illumination for $120 \mathrm{~min}$. Based on these results, we selected PET as a final material for fabricating a microfluidic optical cell.

\section{Detection of rhodamine B inside the microfluidic optical cell}

Rhodamine B has been studied as a sensor chemical because of its strong UV-vis absorption property and its color. Here, we detected rhodamine $\mathrm{B}$, which is supposed to have a UV-vis absorption peak at $550 \mathrm{~nm},{ }^{36-38}$ using the microfluidic optical cell imprint-molded on PET. A simple chamber was formed on PET (length, $8 \mathrm{~mm}$; width, $8 \mathrm{~mm}$; depth, $0.5 \mathrm{~mm}$ ) and bonded with a flat PET via thermal bonding at $60^{\circ} \mathrm{C}$ under $0.1 \mathrm{MPa}$ for $20 \mathrm{~min}$, assisted by the amine-PDMS linker, ${ }^{31}$ which was also used as an anti-adhesion coating material in this study. After bonding, the assembly was pasted onto a solid sample holder, which had an empty hole through which light could pass (Fig. 5(a)).

Figures 5(b) and 5(c) show the absorption intensities of the rhodamine B solution measured inside the imprint-molded PET and micromilled PET, respectively, as a function of the wavelength and the concentration, when concentrations were varied from $3.3 \times 10^{-6}$ to $2.1 \times 10^{-4} \mathrm{~mol} \mathrm{~L}^{-1}$. The intensities of the peaks obtained from the imprint-molded PET were much higher for the same concentration of rhodamine B because the much smoother surface prevented UV scattering inside the microchannel. For example, when the concentration of rhodamine $\mathrm{B}$ was $2.1 \times 10^{-4} \mathrm{~mol} \mathrm{~L}^{-1}$, the measured absorption intensity was approximately 4.3 -fold higher inside the imprintmolded PET than inside the micromilled PET. The adjusted R-square value was 0.999 for the imprint-molded PET (Fig. 5(b)), whereas that of the micromilled PET was 0.804 (Fig. 5(c)). These results confirmed that the microchannel fabricated on PET by imprint molding significantly improved the detection sensitivity.
Detection of copper ions inside the microfluidic optical cell

Figure 6 shows the results of copper ion detection performed using the PET microfluidic optical cell. Different concentrations of copper ion solutions were formulated by dissolving copper(II) sulfate pentahydrate $(>98.0 \%)$ in distilled water. As shown in Fig. 6, the absorption maximum for the copper ions was approximately $790 \mathrm{~nm}$, which corresponds to that of the free hydrated copper species, $\left[\mathrm{Cu}(\mathrm{II})\left(\mathrm{H}_{2} \mathrm{O}\right)_{6}\right]^{2+}{ }^{39-42}$ The absorption intensities for different copper ions measured inside the imprintmolded PET microfluidic optical cell were relatively higher than those measured inside the micromilled PET microfluidic optical cell when detecting the same concentration of copper ions. When the concentration of copper ion was lower than $0.1 \mathrm{~mol} \mathrm{~L}^{-1}$, the concentration difference was difficult to distinguish when using the micromilled optical cell. From these results, we confirmed the potential of using the imprint-molded thermoplastic microchannel as an absorption detector, which could further be functionally integrated with other microfluidic components to realize a totally integrated sample-in-solutionout microdevice.

\section{Conclusions}

In this paper, we report on a new method for fabricating microchannels with improved roughness via imprint molding. This method for replicating microchannels is highly effective regardless of the type of thermoplastic used. Because the method simply requires heating the thermoplastics at temperatures slightly lower than the glass transition temperature of the substrate, and because the mold is recyclable, this method results in reproducible roughness with a minimal fluctuation, as compared to the micromilling process. By using this method, smooth PET microchannels were readily fabricated and used for fabricating a microfluidic optical cell for the detection of rhodamine B and copper ions. This could pave the way for constructing microscale absorption cells as detection modules, allowing for the eventual realization of fully integrated microdevices, from the sample pretreatment process to the readout of the final target product in one continuous and seamless operation. 


\section{Acknowledgements}

This research was supported by Basic Science Research Program through the National Research Foundation of Korea (NRF) funded by the Ministry of Science, ICT \& Future Planning (2014R1A1A3051319), and the Public Welfare \& Safety Research program through the National Research Foundation of Korea (NRF) funded by the Ministry of Science, ICT \& Future Planning (NRF-2012M3A2A1051681).

\section{References}

1. C. F. A. Floquet, V. J. Sieben, A. Milani, E. P. Joly, I. R. G. Ogilvie, H. Morgan, and M. C. Mowlem, Talanta, 2011, 84, 235.

2. D. J. Harrison, K. Fluri, K. Seiler, Z. Fan, C. S. Effenhauser, and A. Manz, Science, 1993, 261, 895.

3. M. Hervás, M. Á. López, and A. Escarpa, Analyst, 2009, 134, 2405.

4. H. Zhao, Z. Li, N. Y. Lee, J. S. Kim, and E.-C. Lee, Curr. Appl. Phys., 2012, 12, 1493.

5. J. Yue, F. H. Falke, J. C. Schouten, and T. A. Nijhuis, Lab Chip, 2013, 13, 4855.

6. A. D. Beaton, V. J. Sieben, C. F. A. Floquet, E. M. Waugh, S. A. K. Bey, I. R. G. Ogilvie, M. C. Mowlem, and H. Morgan, Sens. Actuators, B, 2011, 156, 1009.

7. C. M. McGraw, S. E. Stitzel, J. Cleary, C. Slater, and D. Diamond, Talanta, 2007, 71, 1180.

8. D. Gao, H. Liu, Y. Jiang, and J.-M. Lin, Lab Chip, 2013, $13,3309$.

9. M. Prudent and H. H. Girault, Analyst, 2009, 134, 2189.

10. J. Lee, S. A. Soper, and K. K. Murray, Analyst, 2009, 134, 2426.

11. I. R. G. Ogilvie, V. J. Sieben, C. F. A. Floquet, R. Zmijan, M. C. Mowlem, and H. Morgan, J. Micromech. Microeng., 2010, 20, 065016 .

12. H. Becker and U. Heim, Sens. Actuators, A, 2000, 83, 130.

13. G.-B. Lee, S.-H. Chen, G.-R. Huang, W.-C. Sung, and Y.-H. Lin, Sens. Actuators, B, 2001, 75, 142.

14. T. Lomas, S. Mongpraneet, A. Wisitsoraat, K. Jaruwongrungsee, A. Sappat, T. Maturos, F. Chevasuvit, and A. Tuantranont, in Proceedings of the Sixth International Conference on Electrical Engineering/Electronics, Computer, Telecommunications and Information Technology (ECTI-CON), 2009, Pattaya, Thailand, 462.

15. J. Kim and X. Xu, J. Laser Appl., 2003, 15, 255.

16. R. Srinivasan and V. Mayne-Banton, Appl. Phys. Lett., 1982, 41, 576.

17. V. Srinivasan, M. A. Smrtic, and S. V. Babu, J. Appl. Phys., 1986, 59, 3861.
18. M. F. Jensen, J. E. McCormack, B. Helbo, L. H. Christensen, T. R. Christensen, and O. Geschke, Lab Chip, 2004, 4, 391.

19. H. H. Tran, K. T. L. Trinh, and N. Y. Lee, J. Chromatogr. B, 2013, 936, 88.

20. J. K. Lee, K.-W. Park, J. C. Choi, H.-R. Kim, and S. H. Kong, J. Micromech. Microeng., 2012, 22, 115028.

21. Y. Huang, S. Liu, W. Yang, and C. Yu, Appl. Surf. Sci., 2010, 256, 1675.

22. L. Tang and N. Y. Lee, Lab Chip, 2010, 10, 1274.

23. X. Gong, W. Wen, and P. Sheng, Langmuir, 2009, 25, 7072.

24. S. S. Liow, L. K. Widjaja, V. T. Lipik, and M. J. M. Abadie, Express Polym. Lett., 2009, 3, 159.

25. N. B. Cramer, S. K. Reddy, H. Lu, T. Cross, R. Raj, and C. N. Bowman, J. Polym. Sci. Pol. Chem., 2004, 42, 1752.

26. B.-S. Chiou and S. A. Khan, Macromolecules, 1997, 30, 7322.

27. B.-S. Chiou, R. J. English, and S. A. Khan, Macromolecules, 1996, 29, 5368.

28. C. Li and G. L. Wilkes, J. Inorg. Organomet. Polym., 1997, 7, 203.

29. C. F. Carlborg, T. Haraldsson, K. Öberg, M. Malkoch, and W. van der Wijingaart, Lab Chip, 2011, 11, 3136.

30. L. Derzsi, P. Jankowski, W. Lisowski, and P. Garstecki, Lab Chip, 2011, 11, 1151.

31. J. Wu and N. Y. Lee, Lab Chip, 2014, 14, 1564.

32. A. Hasaninejad, A. Zare, A. Khalafi-Nezhad, H. Sharghi, A. R. Moosavizare, and A. Parhami, J. Chil. Chem. Soc., 2008, 53, 1663.

33. K. A. Whitehead, G. Sahay, G. Z. Li, K. T. Love, C. A. Alabi, M. Ma, C. Zurenko, W. Querbes, R. S. Langer, and D. G. Anderson, Mol. Ther, 2011, 19, 1688.

34. A. Southan, M. Mateescu, V. Hagel, M. Bach, C. Schuh, C. Kleinhans, P. J. Kluger, S. Tussetschläger, I. Nuss, T. Haraszti, S. V. Wegner, J. P. Spatz, H. Boehm, S. Laschat, and G. E. M. Tovar, Macromol. Chem. Phys., 2013, 214 , 1865.

35. N. Y. Lee, Colloid Surf., B, 2013, 111, 313 .

36. H. Tokudome, Y. Yamada, S. Sonezaki, H. Ishikawa, M. Bekki, K. Kanehira, and M. Miyauchi, Appl. Phys. Lett., 2005, 87, 213901.

37. Z. Cheng, S. Wang, Q. Wang, and B. Geng, CrystEngComm, 2010, 12, 144.

38. N. Megouda, Y. Cofininier, S. Szunerits, T. Hadjersi, O. ElKechai, and R. Boukherroub, Chem. Commun., 2011, 47, 991.

39. J. Bjerrum, C. J. Ballhausen, and C. K. Jørgensen, Acta Chem. Scand., 1954, 8, 1275.

40. M. M. Hassanien, M. H. Abdel-Rhman, and A. A. El-Asmy, Transit. Met. Chem., 2007, 32, 1025.

41. C. K. Jørgensen, Acta Chem. Scand., 1956, 10, 887.

42. R. Song, K. M. Kim, and Y. S. Sohn, Inorg. Chem., 2003, 42,821 . 